were interrogated to identify pathogens. A host transcriptomic MLC was developed from human RNA transcripts using 70 cases. The MLC and mNGS reporting thresholds were then tested on 108 blinded cases within the cohort.

Results mNGS was $75 \%$ concordant(27/36) for detecting TB in definite TBM cases and 59\% concordant(30/51) in definite/ probable TBM combined. $3 \mathrm{~TB}$ and 3 non-TB pathogens were detected in the probable TBM group. In the possible TBM/ indeterminant groups, mNGS identified 3 cases of TBM and 17 other pathogens. The combined mNGS and host-MLC displayed $83.3 \%(5 / 6)$ sensitivity, $86.8 \%(59 / 68)$ specificity, with an area under the ROC curve of $0.83(\mathrm{p}=0.009)$.

Conclusion mNGS identified an array of infectious TBM mimics, including many treatable and vaccine preventable pathogens. mNGS was $75 \%$ concordant with definite TBM. We further enhanced the sensitivity of the CSF mNGS assay by developing the first CSF-based host MLC to discriminate between TBM and its mimics

\section{PREVALENCE OF MRI SIGNS OF INTRACRANIAL HYPERTENSION AND THEIR ASSOCIATION WITH PAPILLEDEMA: A PROSPECTIVE STUDY USING OCULAR FUNDUS PHOTOGRAPHY}

\footnotetext{
1,2,3Benson S Chen, ${ }^{1}$ Benjamin I Meyer, ${ }^{1}$ Amit M Saindane, 'Beau B Bruce, ${ }^{1}$ Nancy J Newman, 'Valérie Biousse. 'Emory University School of Medicine, Atlanta, GA, USA; ${ }^{2}$ Department of Clinical Neurosciences, University of Cambridge, Cambridge, UK; ${ }^{3}$ Cambridge Eye Unit, Addenbrooke's Hospital, Cambridge, UK
}

\subsection{6/bmino-2021-ANZAN.39}

Objectives MRI signs of intracranial hypertension (MRI-IH) are classically associated with idiopathic intracranial hypertension (IIH), but also detected in asymptomatic individuals without papilledema. The objective of this study was to determine the prevalence of MRI-IH in consecutive outpatients undergoing brain MRI for any clinical indication, and explore their association with papilledema.

Methods Prospective cross-sectional observational study of outpatients undergoing brain MRI, with ocular fundus photographs taken concurrently. Radiologic studies were analyzed for MRI-IH. Univariate analysis with Fisher's exact test or ttest was performed.

Results Of 296 patients included, the most common indication for MRI was surveillance of a brain neoplasm (27.7\%). Investigation of headaches $(8.8 \%)$ or disorders of raised ICP (1.4\%) were uncommon. At least one MRI-IH was present in $49 \%$ of patients [empty sella (33.1\%), enlarged Meckel's cave $(15.9 \%)$, increased peri-optic CSF $(10.8 \%)$, optic nerve tortuosity (7.8\%), scleral flattening $(0.7 \%)$, cephaloceles $(1.4 \%)$. Bilateral transverse venous sinus stenosis (TVSS) was present in $3.0 \%$ of 198 patients. Five patients $(1.7 \%)$ had papilledema. Compared to patients without papilledema, those with papilledema had significantly higher BMI and prior history of IIH, and increased prevalence of empty sella, optic nerve tortuosity, and TVSS on MRI. The prevalence of papilledema increased from $2.8 \%$ among patients with at least one MRI-IH to $40 \%$ among patients with four or more MRI-IH.

Conclusion MRI-IH are common in patients undergoing brain MRI, but rarely associated with papilledema. The management of patients with incidentally detected MRI-IH likely does not require systematic lumbar puncture unless concerning symptoms or papilledema are present.

\section{THE QUEST TO REDUCE STROKE TREATMENT DELAYS AT A MELBOURNE METROPOLITAN PRIMARY STROKE CENTRE OVER THE LAST TWO DECADES}

1,2 2 eter SW Park, ${ }^{1}$ Tanya Frost, ${ }^{1}$ Peter SY Tan, ${ }^{1}$ Joseph Wong, ${ }^{2}$ Alun Pope, ${ }^{1,2}$ Helen M Dewey, ${ }^{1,2}$ Philip MC Choi. 'Department of Neurosciences, Box Hill Hospital, Eastern Health, Box Hill, VIC, Australia; ' 2 Eastern Health Clinical School, Faculty of Medicine, Nursing and Health Sciences, Box Hill, VIC, Australia

\subsection{6/bmino-2021-ANZAN.40}

Objectives To examine door-to-needle time (DNT) trends and the impact of continuous quality improvement initiatives since thrombolysis became standard of care in Australian metropolitan setting for eligible acute ischaemic stroke patients.

Methods Single-centre retrospective cohort study of consecutive patients treated with thrombolysis at high volume primary stroke centre from January 2003 to December 2019. Primary outcomes are DNT, and annual number of 'Code Stroke' activations and stroke admissions. Regression modelling for potential factors associated with DNT.

Results 1,250 patients were treated with thrombolysis over 17 years; 54\% were male with a median age of 76 (interquartile ranges [IQR], 66-83). Median DNT fluctuated between 70 to 93 minutes (IQR, 55-95 to 82-120) from 2003 to 2012, reaching 60 minutes in 2013 and nadir of 47 minutes in 2014. Median DNT then decreased from 58 minutes in 2015 to 51 minutes in 2019 with progressive tightening of IQR (46-78 to 40-62). Number of patients treated within 60 minutes of hospital arrival was less than $30 \%$ between 2003-2012. This rose to an average of $63 \%$ during 2015-2018 and $71 \%$ in 2019. From 2015 to 2019 , per annum number of 'Code Stroke' activations increased from 940 to 1300 while stroke admissions plateaued at 750 . 'Direct-to-CT' protocol and acute stroke presence were two modifiable workflow factors independently associated with faster DNT $(\mathrm{P}<0.001)$.

Conclusion Targeted quality improvement initiatives are key to reducing treatment delays in the Australian metropolitan setting. Relative stagnation in DNT improvement is concerning and needs further investigation.

\section{COGNITIVE IMPAIRMENT IN LATE ONSET EPILEPSY}

Xin Zhang, Armin Nikpour, Rebekah Ahmed. Royal Prince Alfred Hospital, Camperdown, NSW, Australia

\subsection{6/bmjno-2021-ANZAN.41}

Objective Late onset, unprovoked epilepsy patients with cognitive impairment can have complex pathophysiology. ${ }^{1}$ Our objective was to study the characteristics and contributors of cognitive impairment in this group; and how patients with dementia could be differentiated from late onset epilepsy patients.

Methods Twenty-six patients with epilepsy, onset after 50 years of age, with new cognitive complaints and 26 patients with clinically diagnosed Alzheimer's Disease (AD) were recruited. These participants had comprehensive neuropsychological and neuroimaging assessments. A subset of 17 participants from the Epilepsy group underwent longitudinal neuropsychological assessment.

Results In the Epilepsy group, the neuropsychological profile of cognitive impairment was consistent with the foci and severity of seizure activity in $46 \%$ of participants; subcortical 
microvascular change in $15 \%$; mood disturbance in $15 \%$; medication in 15\%; alcohol in $4 \%$ and $\mathrm{AD}$ in $4 \%$. Compared with the Epilepsy group, the $\mathrm{AD}$ group had a lower Addenbrookes Cognitive Examination III (ACE-III) score $(79.3 \pm 10.8$ versus $87.5 \pm 6.5, p=0.01)$; specifically in the attention, memory and visuospatial subdomains $(p=0.004, p=0.002$ and $p=0.02)$ but not fluency and language subdomains $(p>0.05)$; and lower scores on additional assessments of naming, visuospatial and executive function ( $p \leq 0.001$ ). The AD group had more abnormal metabolism in the temporal, parietal and occipital lobes than the Epilepsy group $(p=0.02, p=0.006$ and $p=0.005$ ).

Conclusion Patients with late onset epilepsy and cognitive complaints rarely have dementia diagnosed at their first neuropsychological assessment and tend to have milder cognitive impairment than patients with $\mathrm{AD}$. The two groups can be differentiated by their neuropsychological and FDG-PET profiles.

\section{REFERENCE}

1. Sen A, Capelli V, Husain M. Cognition and dementia in older patients with epilepsy. Brain 2018;141(6):1592-1608.

\section{Poster abstracts}

\section{A CASE OF ISOLATED MUSCULOCUTANEOUS NERVE INJURY FOLLOWING SKYDIVING SIMULATION}

Oshi Swarup, Belinda Cruse, Daniela Leupold, John King. Department of Neurology, Royal Melbourne Hospital, Parkville, Melbourne, VIC, Australia

\subsection{6/bmjno-2021-ANZAN.42}

Background Isolated musculocutaneous nerve injuries are rare, and mostly iatrogenic or traumatic.

Case Presentation We present a case of isolated musculocutaneous neurapraxia in an otherwise well young woman following uncomplicated simulated skydiving.

Management and Outcome While the injury was quite debilitating, complete neurological recovery occurred within two months without any intervention.

Discussion This case illustrates a rare pattern of neurological injury, caused by a recreational activity growing in popularity. The pattern of injury mimics that of an upper trunk brachial plexopathy or $\mathrm{C} 5 / 6$ radiculopathy. Increased awareness of the injury avoids misdiagnosis and affords the opportunity for prevention.

\section{NEUROLOGICAL MANIFESTATIONS IN RHEUMATOLOGICAL DISEASE: A CASE SERIES I}

Eileen JMc Manus, Douglas White, Alan Doube, Jan Schepel, Matthew Phillips, Kamal Solanki. WDHB, Hamilton, Waikato, New Zealand

\subsection{6/bmjno-2021-ANZAN.43}

Objective Rheumatology encompasses a broad range of multisystemic, autoimmune and inflammatory disorders. Neurological manifestations of these diseases are not uncommon. Neurological findings may predate rheumatological findings or may emerge months to years post initial diagnosis. Rheumatological diseases presenting as neurological syndromes can cause diagnostic challenges.
Methods/Results We present a range of rheumatological cases with unusual neurological presentations that demonstrate this point including; C2-C3 facet arthropathy in Diffuse Scleroderma, Granulomatosis with polyangiitis manifesting with craniofacial involvement, pseudo vasculitis associated cerebrovascular events, SAPHO syndrome with a thoracic syrinx, Neuro- Bechet's vasculitis with tumour-like CNS lesions, Platybasia in Paget's disease and others.

Conclusions Familiarity with the neurological manifestations of rheumatologic diseases is important for both rapid diagnosis and appropriate intervention.

\section{METABOLIC SYNDROME IN A NEW ZEALAND GLIOBLASTOMA COHORT 2005-2020: A RETROSPECTIVE ANALYSIS AND REVIEW OF THE LITERATURE}

${ }^{1}$ Eileen JMc Manus, ${ }^{1,2}$ Christopher Frampton, ${ }^{1}$ Alvin Tan, ${ }^{1}$ Matthew Phillips. ${ }^{1}$ WDHB, Hamilton, Waikato, New Zealand; '2Department of Medicine, University of Otago, Christchurch, New Zealand

\subsection{6/bmino-2021-ANZAN.44}

Background Glioblastoma (GBM) is an aggressive form of glioma. Even with standard-of-care Stupp protocol (surgery, radiotherapy, and temozolomide), median overall survival is only 10-12 months in population-based studies. Metabolic reprogramming is a hallmark of glioblastoma, with energy metabolism aberrantly geared towards aerobic fermentation. The prevalence of metabolic syndrome is $16 \%$ in the general $\mathrm{NZ}$ population and $32 \%$ in the Maori population.

Objectives We aimed to determine 1) if metabolic syndrome was more prevalent in our GBM cohort compared to general NZ population 2) if metabolic syndrome was associated with worse overall survival in GBM 3) if ethnicity influenced survival outcomes.

Methods We performed a retrospective analysis of 170 patients diagnosed and treated for GBM between 2005-2020 in one institution. Clinical and biochemical data relating to metabolic syndrome were collected. Overall survival was determined from the date of initial a surgical diagnosis to the date of death or data acquisition.

Results $18.2 \%$ of GBM patients met the criteria for metabolic syndrome, $27.7 \%$ of Maori and $16.1 \%$ of European New Zealanders. Patients with metabolic syndrome had statistically significant worse overall survival compared to those patients without metabolic syndrome regardless of treatment [mean 9.7 vs 18.4 months] $\mathrm{p}=0.016(\mathrm{p} \leq 0.05)$. Power was too low to comment on the prevalence of metabolic syndrome or ethnicity.

Conclusion Our study demonstrates that metabolic syndrome is associated with statistically significant poorer outcome in GBM patients. Consequently, this data will provide a control group for our current prospective study investigating the antineoplastic effects of metabolic therapies in GBM.

\section{MULTIPLE CRANIAL NEUROPATHIES IN A PATIENT WITH SYPHILITIC MENINGITIS}

${ }^{1}$ Melissa Chu, 'Shejil Kumar, 1,2Jonathan Sturm. 'Gosford Hospital, Gosford, NSW, Australia; ${ }^{2}$ Neurology, University of Newcastle, Newcastle, NSW, Australia

10.1136/bmjno-2021-ANZAN.45 